DGOR-Jahrestagung 1983

21.-23. September - Mannheim

Auskunft: Prof. Dr. Ch. Schneeweiß

Universität Mannheim

Lehrstuhl für Betriebswirtschaftslehre

und Unternehmensforschung

Schloß

D-6800 Mannheim 1, F.R. Germany

13. Jahrestagung der Gesellschaft für Informatik

3.-7. Oktober 1983 - Hamburg, FRG

Auskunft: I. Kupka

FB Informatik der Universität Hamburg

Schliuterstraße 70

D-2000 Hamburg 13

Angewandte Wirtschafts- und Sozialkybernetik

Jahrestagung 1983 der Gesellschaft für Wirtschafts- und Sozialkybernetik 18.-19. November 1983 - Marburg

Auskunft: Prof. Dr. Bernd Schiemenz Fachbereich Wirtschaftswissenschaften der Philipps-Universität Marburg

Universitätsstraße 25

D-3550 Marburg 1

Seventh European Meeting on Cybernetics and Systems Research 24.-27. April 1984 - Wien, Austria
Auskunft: Prof. Robert Trappl

Dept. of Medical Cybernetics

University of Vienna

Freyung 2/6, A-1010 Vienna, Austria

26th International Meeting of The Institute of Management Science

18.-20. Juni 1984 - Copenhagen, Denmark

Auskunft: Julie Eldridge, TIMS

146 Westminster Street

Providence, Rhode Island 02903, USA

Third International Conference on System Science in Health Care 16.-20. Juli 1984 - München, F. R. Germany

Auskunft: Dr. Rolf Engelbrecht

Chairman Organizing Committee

c/o MEDIS Institut der GSF - München

Ingolstädter Landstraße 1

D-8042 Neuherberg, F. R. Germany

COMPSTAT' 84

27.-31. August 1984 - Praha, Czechoslovakia

Auskunft: Dr. Th. Havranek

Czechoslovak Academy of Sciences

Center of Biomathematics

Budějovická 1083

CS.14220 Praha, CSSR

\title{
DGOR-Seminare im 2. Halbjahr 1983
}

Für das 2. Halbjahr 1983 (und evtl. Anfang 1984) sind folgende Seminare geplant:

K.-P. Schuster, Hamburg und Prof. Dr. V. Steinecke, Dortmund

Prof. Dr. H. Krallmann, Berlin

Prof. Dr. H. Krallmann, Berlin

Stichprobeninventur 23./24. 6. 1983 in Köln und 10./11. 11. 1983 in München

Unternehmensplanung und Informationstechnologien

Entscheidungsunterstützungsverfahren

Prof. Dr. H. Müller-Merbach, Entwurf von EDV-Sollkonzepten Darmstadt

Prof. Dr. P. Hamann, Bochum
Dr. U. Winand, Köln

Prof. Dr. J. Schwarze, Braunschweig
Petri-Netze

Prognoseverfahren in der Praxis 24./25. 11. 1983

Für Einzelheiten zu den Seminaren (auch bei Redaktionsschluß noch nicht feststehende Termine und Orte), Anmeldungen und Rückfragen wenden Sie sich bitte an unseren Veranstalter:

mbp

Mathematischer Beratungs-

und Programmierungsdienst $\mathrm{GmbH}$

Methoden und Training

Semerteichstraße 47

4600 Dortmund 1

Telefon (0231) 4348-0

Telex $8277198 \mathrm{mbp} \mathrm{d}$ 